\title{
Survival in Mesenchymal Chondrosarcoma Varies Based on Age and Tumor Location: A Survival Analysis of the SEER Database
}

\author{
Brian A. Schneiderman MD, Stephanie A. Kliethermes PhD, Lukas M. Nystrom MD
}

Published online: 14 March 2016

(C) The Association of Bone and Joint Surgeons (B) 2016

\begin{abstract}
Background Studies suggest that mesenchymal chondrosarcoma is associated with a poorer prognosis and a higher proportion of extraskeletal tumors than conventional chondrosarcoma. However, these investigations have been small heterogeneous cohorts, limiting analysis of prognostic factors.

Questions/purposes (1) What is the 5- and 10-year survival rate of patients diagnosed with mesenchymal chondrosarcoma? (2) What is the effect of demographic and tumor characteristics on survival in patients with mesenchymal chondrosarcoma?

Methods The Surveillance, Epidemiology, and End Results (SEER) database was used to identify all patients diagnosed with mesenchymal chondrosarcoma from 1973 to 2011. SEER reports survival data on over 8.2 million patients with cancer and has attained $98 \%$ completeness in reporting. Using variables within the database, this study designated each patient's tumor as skeletal or extraskeletal
\end{abstract}

Each author certifies that he or she, or a member of his or her immediate family, has no funding or commercial associations (eg, consultancies, stock ownership, equity interest, patent/licensing arrangements, etc.) that might pose a conflict of interest in connection with the submitted article.

All ICMJE Conflict of Interest Forms for authors and Clinical Orthopaedics and Related Research $\mathbb{R}$ editors and board members are on file with the publication and can be viewed on request.

This work was performed at Loyola University Chicago, Stritch School of Medicine, Maywood, IL, USA.

B. A. Schneiderman

Harbor-UCLA Medical Center, Los Angeles, CA, USA

B. A. Schneiderman, S. A. Kliethermes, L. M. Nystrom ( $\square)$ Stritch School of Medicine, Loyola University Chicago, 2160 South First Avenue, Maywood, IL 60153, USA

e-mail: lnystrom@luc.edu and cranial, axial, or appendicular, respectively. Overall survival (OS) was determined for the entire series as well as each group. Median survival was calculated using KaplanMeier methods. Cox proportional hazards regression was used to determine whether demographic and tumor variables affected survival. Two hundred five patients with mesenchymal chondrosarcoma were identified, including 82 (40\%) skeletal and $123(60 \%)$ extraskeletal.

Results OS for the entire series was 51\% (95\% confidence interval [CI], $43 \%-58 \%)$ and $43 \%(95 \% \mathrm{CI}, 35 \%-51 \%)$ at 5 and 10 years, respectively. No difference in OS was detected between extraskeletal and skeletal tumors. KaplanMeier analyses showed OS was worse for tumors in axial locations compared with appendicular and cranial locations. Appendicular tumors demonstrated an OS of $50 \%$ (95\% CI, $36 \%-63 \%)$ at 5 years and $39 \%(95 \% \mathrm{CI}, 26 \%-52 \%)$ at 10 years. OS for axial tumors was 37\% (95\% CI, 25\%-49\%) and $31 \%$ (95\% CI, 20\%-43\%), whereas it was $74 \%$ (95\% CI, 59\%-84\%) and 67\% (95\% CI, 50\%-79\%) for cranial tumors at 5 and 10 years, respectively. When controlling for age, sex, tumor origin, and tumor location, the presence of metastasis (hazard ratio [HR], 12.38; 95\% CI, 5.75-26.65; $\mathrm{p}$ $<0.001)$ and $1-\mathrm{cm}$ size increase (HR, 1.16; 95\% CI, 1.09$1.23 ; \mathrm{p}<0.001)$ were both independently associated with an increased risk of death. Tumor location showed different behaviors depending on patient age. In comparison to cranial tumors at age 20 years, the HR was $5.56(95 \% \mathrm{CI}$, $1.47-21.05 ; \mathrm{p}=0.01)$ for axial tumors and $6.26(95 \% \mathrm{CI}$, $1.54-25.42 ; \mathrm{p}=0.01$ ) for appendicular tumors. At age 60 years, those ratios were $0.10(95 \% \mathrm{CI}, 0.02-0.55 ; \mathrm{p}=0.01)$ and 0.14 (95\% CI, 0.04-0.58; $\mathrm{p}=0.01$ ), respectively.

Conclusions Our data suggest that extraskeletal tumors are more common than previously reported; however, this factor does not have clear prognostic value. Presence of metastatic disease and increased tumor size are the main 
predictors of poor survival outcome. Cranial tumors appear to have a different clinical behavior with our data suggesting better overall survival in young patients (compared with axial and appendicular locations) and a worse survival outcome in older patients.

Level of Evidence Level IV, prognostic study.

\section{Introduction}

Mesenchymal chondrosarcoma is a rare malignancy that represents $2 \%$ to $9 \%$ of all chondrosarcomas [17]. It arises most commonly in the second and third decades of life [1, $3,8,14]$. It has been associated with late recurrence and metastasis with reports of tumors recurring over 20 years from initiation of primary treatment. In comparison to conventional chondrosarcoma, which has a 10-year overall survival rate of $60 \%$ to $70 \%[6,7,9]$, the mesenchymal subtype is believed to portend a worse prognosis [2, 14, 17]. The 10-year survivorship of mesenchymal chondrosarcoma has been previously reported as ranging from $20 \%$ to $67 \%[3,8,11,14,15,17,18,21]$ with cohorts comprised of head and neck tumors or of pediatric patients representing the upper end of this spectrum [4, 16, 23]. The disease recently was reported to have a median event-free survival of 57 months [25]. Additionally when compared with the $<1 \%$ occurrence of extraskeletal classic chondrosarcoma, mesenchymal chondrosarcoma is thought to have a higher proportion of tumors arising in extraskeletal locations [4, 15]. Extraskeletal mesenchymal chondrosarcoma has been reported to occur in $14 \%$ to $73 \%$ of patients $[4,8,14,15]$ with the largest series reporting that $39 \%$ had extraskeletal tumors [18].

However, as a result of small heterogeneous cohorts previously reported on this rare disease, it has been difficult to achieve a consensus regarding patient and tumor characteristics that affect prognosis. Tumor grade, extent of surgical margins, and the presence of distant disease have all been associated with worse outcomes in conventional chondrosarcoma [9]. However, analysis of the effects of these variables on the prognosis of mesenchymal chondrosarcoma has been limited, and differences in survival between skeletal and extraskeletal mesenchymal chondrosarcoma have not been well defined [2, 8, 11]. Identification of the prognostic implications of disease location has not been completed. Although cranial origin has been associated with a more favorable prognosis, little information is available regarding survival when considering only tumors in axial or appendicular locations [8, 15, 16, 23].

In designing this investigation, we therefore asked: (1) What is the 5- and 10-year survival rate of patients diagnosed with mesenchymal chondrosarcoma? (2) What is the effect of demographic and tumor characteristics such as skeletal origin (skeletal or extraskeletal), primary location of disease (cranial, axial, or appendicular), distant metastasis, tumor size, American Joint Committee on Cancer (AJCC) stage [5], grade, age, and gender on survival in patients with mesenchymal chondrosarcoma?

\section{Materials and Methods}

This investigation was designed as a retrospective review of mesenchymal chondrosarcoma using the Surveillance, Epidemiology, and End Results (SEER) database. SEER reports incidence, population, and survival data on over 8.2 million patients with cancer collected from 18 registries across the nation from the years 1973 to 2011 [19]. For each individual patient with cancer, data are collected regarding demographics, cancer characteristics, and behavior at the time of diagnosis; treatment within 4 months of diagnosis; and patient survival and cause of death, if applicable [10, 19, 21].

As a result of the National Cancer Act of 1971, SEER was founded as an instrument to collect and analyze cancer data in efforts to better understand the incidence, prevalence, and response to treatment of cancer and its subtypes in the United States [10, 24]. The database began as a small group of regional and statewide registries that now includes 18 registries across the country, which in 2010 collectively accounted for $28 \%$ of the US population. Each registry accounts for roughly 100,000 to 21 million individuals with a median population served among all 18 sites of approximately 3.6 million people [19].

Within its geographic area, a registry procures all cancer data on its regional residents from providers, hospitals, and other health services and provides this information to the National Cancer Institute to be included in the database. According to SEER, inclusion of specific registries was based on their generalizable population demographics and ability to maintain high standards in cancer reporting. Age and sex characteristics of the SEER population are comparable to that of the United States; however, SEER data may account for a more urban and affluent population in comparison to the United States as a whole [20, 24].

SEER data are considered robust and valid [10, 12, 13, 19, 24]. There is $98 \%$ completeness in reporting patients with cancer within 22 months of diagnosis across all cancer sites, and annual audits of subsamples of data are issued to evaluate and monitor accuracy [12, 24]. Survival followup is pursued by active and passive methods by each registry for all patients. Although SEER requires that cancer registries must meet or exceed a $95 \%$ successful followup rate, this rate was found to approach $99 \%$ on review of data 
from 1975 to 2012 [13]. This suggests that even if a patient continues treatment at a location outside the region of a registry, there is still high reporting of survival followup. As a result of the rarity of mesenchymal chondrosarcoma and its poor prognosis suggested in previous works, SEER patients of all followup durations were deemed valuable to include in the present study.

The November 2013 release of the SEER data was used to identify all patients diagnosed with mesenchymal chondrosarcoma from 1973 to 2011. Demographic variables for all patients were recorded including age at diagnosis, year of diagnosis, sex, race, and geographic region. Tumor characteristics were extracted from available modifiers and recorded including location, presence of metastatic disease at diagnosis, histological grade, AJCC stage, tumor size (maximal dimension in millimeters), current status (no evidence of disease, alive with disease, died of disease, died of other causes), and length of followup or survival (in months).

As a result of omissions in reporting, data regarding metastasis, grade, AJCC stage, and size were not available for $19(9 \%), 119(58 \%), 148(72 \%)$, and $87(42 \%)$ of the 205 patients observed in this study, respectively.

Because the SEER database does not specifically identify skeletal or extraskeletal origin, the "primary site-labeled" variable in SEER was used to categorize skeletal or extraskeletal origin and cranial, axial, or appendicular tumor location. Each observation was reviewed and categorized by each author independently. Classification was not difficult, because most "primary site" designations clearly referred to either a bony or soft tissue site of origin. When these data were difficult to interpret (10 cases, $<5 \%$ of the data set), categorization occurred after discussion and consensus between authors as to the meaning of the reported data.

\section{Statistical Analysis}

Overall (OS) and disease-specific survival (DSS) was determined for the entire series as well as by tumor site and locations (skeletal, extraskeletal, axial, cranial, and appendicular).

Median, 5-year, and 10-year survival were each calculated using Kaplan-Meier methods (eg, log-rank tests) with Sidak corrections used for post hoc comparisons. Survival was defined as the time from initial diagnosis to the time of patient death or the last date of followup. Cox proportional hazards regression was used to determine whether demographic and tumor characteristic variables affected survival. Specifically, age, sex, skeletal or extraskeletal origin, tumor size, metastatic disease, and axial cranial or appendicular location were examined as well as all two-way interactions. The final model was chosen based on the Akaike Information Criterion and met proportional hazards assumptions. Statistical significance was assessed at $\alpha \leq 0.05$ and SAS Version 9.4 (SAS Institute, Cary, NC, USA) was used for all analyses.

Two hundred seven individuals with mesenchymal chondrosarcoma were identified in the SEER database, and two were excluded as a result of incomplete data regarding site of origin, resulting in 205 patients for analysis. There were $114(56 \%)$ men and 91 (44\%) women. Exact tumor location was not documented in six patients, all of which were of skeletal origin. The mean age of all 205 patients was 37 years $(S D=20)$ with $44 \%$ of these patients in their second or third decades of life at the time of diagnosis. Of 205 patients reviewed, $82(40 \%)$ presented with a skeletal tumor, and $123(60 \%)$ presented with an extraskeletal tumor. Univariable Cox proportional hazards models of grade $(\mathrm{N}=78)$ and AJCC stage $(\mathrm{N}=51)$ suggest no association between grade and OS $(p=0.76)$; however, an increased hazard of death was identified for more advanced stages of tumors $(p<0.001)$. Specifically, the risk of dying for individuals with Stage IV tumors is 9.01 times greater than those with Stage I tumors (95\% CI, 1.18-68.87; p = 0.03 ). As a result of small sample sizes, neither variable was considered for multivariable analysis.

As a result of missing data (primarily tumor size), 104 patients were retained for multivariable analyses.

Survival across all individuals ranged from 0 to 240 months. Mean followup was 129 months (SD 8 months). Survival outcomes were available for all patients within this data set at the time of data collection.

\section{Results}

Survivorship of Patients With Mesenchymal Chondrosarcoma

Overall survival for the entire series was $51 \%$ (95\% confidence interval [CI], 43\%-58\%) and 43\% (95\% CI, 35\%$51 \%$ ) at 5 and 10 years, respectively (Fig. 1; Table 1). The median OS was 64 months (95\% CI, 40-122 months). The median DSS was 68 months (95\% CI, 60-76 months).

\section{Factors Associated With Survivorship}

No difference was detected in 10-year OS by skeletal (41\%; 95\% CI, 29\%-53\%) or extraskeletal origin (44\%; 95\% CI, 31\%-52\%) (Table 1). When controlling for patient age, gender, anatomic location, tumor size, and presence of metastasis, there was no difference with the numbers available (Table 2).

Five-year survival, 10-year survival, and median survival were lower in axial locations $(37 \%, 95 \%$ CI, 25\%$49 \%$; 31\%, 95\% CI, 20\%-43\%; 30 months, 95\% CI, 20-59 


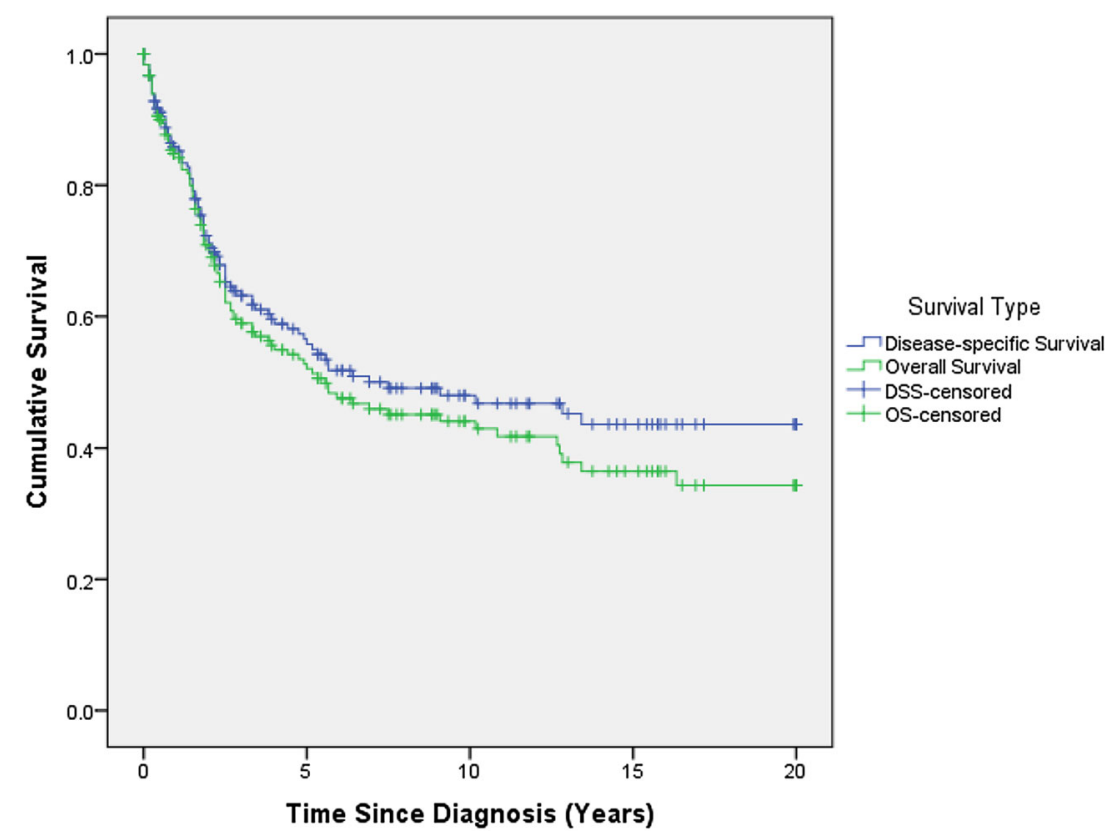

Fig. 1 This figure depicts the Kaplan-Meier curve for OS and DSS for all patients in the entire series.

Table 1. Five- and 10-year overall and median survival stratified by disease location*

\begin{tabular}{llll}
\hline Disease location & 5 -year $(95 \% \mathrm{CI})$ & 10 -year $(95 \% \mathrm{CI})$ & Median months $(95 \% \mathrm{CI})$ \\
\hline $\begin{array}{l}\text { Entire series } \\
\text { Location }\end{array}$ & $51 \%(43 \%-58 \%)$ & $43 \%(35 \%-51 \%)$ & $64(40-122)$ \\
$\quad$ Appendicular & $50 \%(36 \%-63 \%)$ & $39 \%(26 \%-52 \%)$ & $64(28-154)$ \\
$\quad$ Axial & $37 \%(25 \%-49 \%)$ & $31 \%(20 \%-43 \%)$ & $30(20-59)$ \\
$\quad$ Cranial & $74 \%(59 \%-84 \%)$ & $67 \%(50 \%-79 \%)$ & $161(122-$ infinity) \\
Site & $49 \%(36 \%-60 \%)$ & $41 \%(29 \%-53 \%)$ & $60(30-130)$ \\
$\quad$ Skeletal & $52 \%(42 \%-62 \%)$ & $44 \%(31 \%-52 \%)$ & $64(32-154)$ \\
Extraskeletal & & & 0.82
\end{tabular}

* Probability values represent Kaplan-Meier log-rank tests for differences in survival by location and site; CI = confidence interval.

Table 2. Tabulated multivariable Cox proportional hazards analysis for mortality in patients with mesenchymal chondrosarcoma*

\begin{tabular}{lllr}
\hline Variable & Hazard ratio & $95 \%$ CI & p value \\
\hline Age at diagnosis & - & - & 0.81 \\
Metastasis & 12.38 & $5.75-26.65$ & $<0.001$ \\
Gender: male & 1.37 & $0.73-2.57$ & 0.33 \\
Location & - & - & 0.002 \\
Site: skeletal & 1.91 & $0.96-3.79$ & 0.06 \\
Size (10-mm increase) & 1.16 & $1.09-1.23$ & $<0.001$ \\
Age*location & - & - & $<0.001$
\end{tabular}

* Variables found significant in univariable log-rank test were included; reference levels include gender = female; site = extraskeletal; and location = appendicular; appropriate hazard ratios for the age*location interaction are specified in Table 3; main effects of variables included in the interaction are not interpretable and therefore not provided; $\mathrm{CI}=$ confidence interval. 
Table 3. Hazard ratios for mortality are presented by tumor anatomic location at different ages

\begin{tabular}{lllll}
\hline Age (years) & Location* & Hazard ratio & $95 \%$ CI & p value \\
\hline 20 & Axial & 5.56 & $1.47-21.05$ & 0.01 \\
& Appendicular & 6.26 & $1.54-25.42$ & 0.01 \\
30 & Axial & 2.23 & $0.78-6.36$ & $0.13-6.79$ \\
& Appendicular & 2.23 & $0.41-1.94$ & 0.82 \\
40 & Axial & 0.89 & $0.27-2.32$ & 0.16 \\
& Appendicular & 0.80 & $0.08-1.04$ & 0.06 \\
50 & Axial & 0.28 & $0.12-1.06$ & 0.06 \\
60 & Appendicular & 0.36 & $0.02-0.55$ \\
\end{tabular}

* All hazard ratios are compared with the cranial location; $\mathrm{CI}=$ confidence interval.

months, respectively) compared with cranial (74\%, 95\% CI, 59\%-84\%; 67\%, 95\% CI, 50\%-79\%; 161 months, $95 \%$ CI, 122-infinity, respectively); however, no differences in 5-year or 10-year survival were detected when comparing axial with appendicular or appendicular with cranial disease (Table 1). When controlling for gender, tissue type, size, and metastasis, the association between OS and disease location differed by age (Tables 2, 3). Although having a better prognosis in younger patients, cranial tumors were noted to carry a graver prognosis in older patients (Table 3). At age 20 years, the hazard of dying from axial and appendicular tumors was 5.56 (95\% CI, $1.47-21.05 ; \mathrm{p}=0.01)$ and 6.26 (95\% CI, 1.54-25.42; $\mathrm{p}=$ 0.01 ) that of cranial tumors. However, by age 60 years, the hazard was $0.1(95 \% \mathrm{CI}, 0.02-0.55 ; \mathrm{p}=0.01)$ and 0.14 (95\% CI, 0.04-0.58; $\mathrm{p}=0.01$ ) for axial and appendicular tumors compared with cranial tumors, respectively, after controlling for gender, tissue type, size, and metastasis.

After controlling for age, gender, extraskeletal/skeletal site, and anatomic location (using multivariable analysis), both metastasis (hazard ratio [HR], 12.38; 95\% CI, 5.7526.65; $\mathrm{p}<0.001)$ and a $1 \mathrm{~cm}$ increase in tumor size (HR, 1.16 ; $95 \%$ CI, $1.09-1.23 ; \mathrm{p}<0.001$ ) were found to be independent predictors of death from disease (Table 2).

\section{Discussion}

Although general trends and features of the clinical behavior of mesenchymal chondrosarcoma have been suggested in prior studies [1, 14, 15, 17, 18], no large series have been available to verify these descriptions as a result of the rarity of this disease. To date, the largest study on mesenchymal chondrosarcoma was performed by Nakashima et al. [18], which presented 111 new patients with mesenchymal chondrosarcoma and reviewed 132 previously reported patients. However, that investigation included survival analysis for only 23 patients and contained limited subgroup analysis. The wide range of tumor and patient characteristics within the small cohorts precludes meaningful survival analysis. We found that mesenchymal chondrosarcoma accounted for $4 \%$ of the 5110 patients with chondrosarcoma within the SEER database, which is consistent with the $2 \%$ to $9 \%$ reported in prior studies $[1,14,18]$. The 5 - and 10- year OS in this series was $51 \%$ and $43 \%$, respectively. We found that $60 \%$ of all tumors occurred in extraskeletal locations. Survival did not differ between extraskeletal and skeletal locations; however, it varied substantially by anatomic location with 5 -year overall survival rates of $74 \%$ for cranial, $50 \%$ appendicular, and $37 \%$ for axial tumor locations.

This study had a number of limitations, most of which are largely attributable to constraints inherent to a database analysis. First, mesenchymal chondrosarcoma is an extremely rare disease and difficult to diagnose. In completing this investigation we had to assume that the histologic diagnosis was accurate. It is possible that other neoplastic processes with a predilection for soft tissue sites could be responsible for the higher number of soft tissue mesenchymal chondrosarcomas noted in this investigation. Incomplete or inconsistent data on some variables prevented investigation of certain factors that may influence patient survival, namely histologic grade and stage. Additionally, some items of interest were not coded for within SEER. SEER does not include information on patient comorbidities or progression of disease over followup. These limitations limit our ability to state which factors are the most important determinants of prognosis in this disease. Previous investigations into the prognostic factors associated with conventional chondrosarcoma have identified that histologic grade and margin status have been directly related to patient survival [9]. Neither histologic grade nor margin status was uniformly reported in this patient cohort. This represents a substantial limitation within the study because the conclusions regarding the 
importance of other demographic and tumor variables may be confounded by the unknown effect of the grade and margin status. Information regarding use of chemotherapy is not available within the database and the period of inclusion (1973-2011) includes a period of many years before the use of chemotherapy for treatment of musculoskeletal neoplasms. Although this information would have undoubtedly provided meaningful data to analyze as part of this investigation, it is not felt to be a glaring deficit because the use of chemotherapy for treatment of this disease remains controversial, even by current standards [2, 8]. Nevertheless, it is impossible to make any statement regarding the efficacy of chemotherapy on survival. Although we were able to identify the presence of metastatic disease for the vast majority of the patients in our cohort, we were unable to analyze the timing of metastases or recurrence over the course of followup because these time variables were not coded for within SEER. The absence of documentation on timing of local or distant recurrence is a major limitation because this disease has a documented high rate of late distant and local recurrence [4, $8,14,18]$.

The OS of this disease has varied widely ranging from $20 \%$ to $67 \%$ [2-4, 8, 11, 14, 16-18, 22, 23]. Recent studies examining mesenchymal chondrosarcoma in pediatric patients or limited to craniofacial sites represent the more favorable end of this spectrum $[4,16,21,23]$. A study from 2014 of 37 patients by Kawaguchi et al. [15] is the largest survivorship series performed on this disease, reporting 5year survival of $51 \%$ and 10 -year survival of $37 \%$. A recent meta-analysis of 107 previously reported patients with mesenchymal chondrosarcoma found an OS at 5 years of $55 \%$ and at 10 years of $44 \%$ as well as an event-free survival at 10 years of $27 \%$ [25]. Our data indicate a similar OS of $51 \%$ and $43 \%$ at 5 and 10 years, respectively. However, our investigation has identified two critical findings: that extraskeletal tumors are actually more common and that OS varies substantially by patient age and tumor location.

A large variation has been reported with regard to the proportion of patients presenting with extraskeletal mesenchymal chondrosarcoma. It has generally been accepted that skeletal tumors account for the majority of mesenchymal chondrosarcoma diagnoses [8, 18, 25]. In a review of 127 individuals with mesenchymal chondrosarcoma by Huvos et al. [14], 78\% were skeletal in origin. In our analysis, which represents data from across the United States, extraskeletal tumors accounted for $60 \%$ of all mesenchymal chondrosarcoma diagnoses, which suggests that extraskeletal mesenchymal chondrosarcoma is more prevalent than previously reported. Few studies have analyzed survival by skeletal and extraskeletal locations. In an analysis of 13 patients with extraskeletal tumors, Hashimoto et al. found a $42.8 \%$ survival rate at 10 years [11], yet in a separate analysis of 26 tumors, Cesari et al. [2] suggested mesenchymal chondrosarcoma of the soft tissues may be a more aggressive disease than its skeletal counterpart with a 10 -year survival rate of $0 \%$ in such tumors compared with $29 \%$ for skeletal tumors. Although the current investigation found extraskeletal tumors to be more common than previously reported, we found no difference in survival between skeletal and extraskeletal tumors. Although the propensity to arise in extraskeletal locations is an interesting feature of mesenchymal chondrosarcoma, it does not appear to carry any prognostic importance.

Survival by anatomic location of chondrosarcoma overall has been examined by Giuffrida et al. [9]; however, subgroup analysis for mesenchymal chondrosarcoma was not performed. Consistent with previous data, patients with tumors affecting craniofacial sites were found to have a more favorable prognosis $[4,16,23]$. Although this finding may seem counterintuitive, we hypothesize this could be related to easier earlier detection of masses in these locations or potentially a uniformly different biologic behavior of tumors affecting these locations. Additionally, we found an interesting relationship between the relative behavior of the tumors in specific anatomic locations and patient age. In the younger patients, the cranial tumors were found to have a markedly improved prognosis compared with axial and appendicular tumor locations. We found that as we monitored the HR for death, this gradually shifted to a markedly worse prognosis in older patients. This finding deserves further exploration to determine what drives this change in clinical behavior. Although mesenchymal chondrosarcoma tumors involving craniofacial sites have been associated with a more favorable prognosis, previous reports of survival did not uniformly exclude this subgroup from analysis $[4,8,16,23,25]$. As a result, the previous understanding of survival in mesenchymal chondrosarcoma may have demonstrated a bias toward an overly optimistic prognosis in noncranial tumors.

Despite the interesting findings related to the increased incidence of extraskeletal tumors and the survival as it relates to location of tumor and patient age, it should be noted that the presence of metastatic disease and the size of the tumor dominated the model as the main predictors of patient mortality. Although metastatic disease [2, 8] has been previously reported as an important predictor of OS, little attention has been given to the role of tumor size. This finding is not necessarily surprising because increased tumor size is likely a representation of a more biologically active neoplasm.

The prognosis of mesenchymal chondrosarcoma is worse in comparison to classical chondrosarcoma variants; however, it seems not to be as dismal as suggested by some previous studies $[1,2,8]$. Extraskeletal tumors were found to be more common than in earlier reports, but the lack of 
difference in survival between extraskeletal and skeletal tumors suggests this factor has little prognostic importance. The presence of metastatic disease and increased tumor size are clear predictors of a worse OS. Cranial tumors demonstrated a change in clinical behavior between young and old patients with a relatively good prognosis in the young population and an increased likelihood of mortality in older patients. Although this study cannot definitively determine whether the differences in OS of mesenchymal chondrosarcoma are the result of certain tumor characteristics, disparities in treatment, or other factors, it does suggest prognosis for each patient with the disease might be more appropriately evaluated in relation to the patient's age and disease location. Future research should be focused on developing substantial patient populations with careful attention to disease location to examine the effects of these factors on survival.

\section{References}

1. Bertoni F, Picci P, Bacchini P, Capanna R, Innao V, Bacci G, Campanacci M. Mesenchymal chondrosarcoma of bone and soft tissues. Cancer. 1983;52:533-541.

2. Cesari M, Bertoni F, Bacchini P, Mercuri M, Palmerini E, Ferrari S. Mesenchymal chondrosarcoma. An analysis of patients treated at a single institution. Tumori. 2007;93:423-427.

3. Dabska M, Huvos AG. Mesenchymal chondrosarcoma in the young. Virchows Arch A Pathol Anat Histopathol. 1982;399:89-104.

4. Dantonello TM, Int-Veen C, Leuschner I, Schuck A, Furtwaengler R, Claviez A, Schneider DT, Klingebiel T, Bielack SS, Koscielniak E. Mesenchymal chondrosarcoma of soft tissues and bone in children, adolescents, and young adults. Cancer. 2008;112:2424-2431.

5. Edge S, Byrd DR, Compton CC, Fritz AG, Greene FL, Trotti A, eds. AJCC Cancer Staging Manual. $7^{\text {th }}$ ed. New York, NY, USA: Springer-Verlag; 2010.

6. Evans HL, Ayala AG, Romsdahl MM. Prognostic factors in chondrosarcoma of bone. Cancer. 1977;40:818-831.

7. Fiorenza A, Abudu A, Grimer RJ, Carter SR, Tillman RM, Ayoub $\mathrm{K}$, Mangham DC, Davies AM. Risk factors for survival and local control in chondrosarcoma of bone. J Bone Joint Surg Br. 2002;84:93-99.

8. Frezza AM, Cesari M, Baumhoer D, Biau D, Bielack S, Campanacci DA, Casanova J, Esler C, Ferrari S, Funovics PT, Gerrand C, Grimer R, Gronchi A, Haffner N, Hecker-Nolting S, Höller S, Jeys L, Jutte P, Leithner A, San-Julian M, Thorkildsen J, Vincenzi B, Windhager R, Whelan J. Mesenchymal chondrosarcoma: prognostic factors and outcome in 113 patients. A European Musculoskeletal Oncology Society study. Eur J Cancer. 2015;51:374-381.

9. Giuffrida AY, Burgueno JE, Koniaris LG, Gutierrez JC, Duncan R, Scully SP. Chondrosarcoma in the United States (1973 to 2003): an analysis of 2890 cases from the SEER database. $J$ Bone Joint Surg Am. 2009;91:1063-1072.

10. Gloeckler Ries LA, Reichman ME, Lewis DR, Hankey BF, Edwards BK. Cancer survival and incidence from the
Surveillance, Epidemiology, and End Results (SEER) program. Oncologist. 2003;8:541-542.

11. Hashimoto N, Ueda T, Joyama S, Araki N, Beppu Y, Tatezaki S, Matsumoto S, Nakanishi K, Tomita Y, Yoshikawa H. Extraskeletal mesenchymal chondrosarcoma: an imaging review of ten new patients. Skeletal Radiol. 2005;34:785-792.

12. Hayat MJ, Howlader N, Reichman ME, Edwards BK. Cancer statistics, trends and multiple primary cancer analyses from the Surveillance, Epidemiology, and End Results (SEER) program. Oncologist. 2007;12:20-37.

13. Howlader N, Noone AM, Krapcho M, Garshell J, Miller D, Altekruse SF, Kosary CL, Yu M, Ruhl J, Tatalovich Z, Mariotto A, Lewis DR, Chen HS, Feuer EJ, Cronin KA, eds. SEER Cancer Statistics Review, 1975-2012. Bethesda, MD, USA: National Cancer Institute. Available at: http://seer.cancer.gov/csr/1975 2012/, based on November 2014 SEER data submission, posted to the SEER website, April 2015. Accessed April 22, 2015.

14. Huvos AG, Rosen G, Dabska M, Marcove RC. Mesenchymal chondrosarcoma: a clinicopathologic analysis of 35 patients with emphasis on treatment. Cancer. 1983;51:1230-1237.

15. Kawaguchi S, Weiss I, Lin P, Huh W, Lewis V. Radiation therapy is associated with fewer recurrences in mesenchymal chondrosarcoma. Clin Orthop Relat Res. 2014;472:856-864.

16. Knott DP, Gannon FH, Thompson LDR. Mesenchymal chondrosarcoma of the sinonasal tract: a clinicopathological study of 13 cases with a review of the literature. Laryngoscope. 2003;113:783-790.

17. Lichtenstein L, Bernstein D. Unusual benign and malignant chondroid tumors of bone. A survey of some mesenchymal cartilage tumors and malignant chondroblastic tumors, including a few multicentric ones, as well as many atypical benign chondroblastomas and chondromyxoid fibromas. Cancer. 1959;12:1142-1157.

18. Nakashima Y, Unni KK, Shives TC, Swee RG, Dahlin DC. Mesenchymal chondrosarcoma of bone and soft tissue. A review of 111 cases. Cancer. 1986;57:2444-2453.

19. National Cancer Institute. Surveillance, Epidemiology, and End Results (SEER) program. 2014. Available at: http://seer.cancer. gov/data. Accessed February 14, 2016.

20. Nattinger AB, McAuliffe TL, Schapira MM. Generalizability of the Surveillance, Epidemiology, and End Results registry population: factors relevant to epidemiologic and health care research. J Clin Epidemiol. 1997;50:939-945.

21. Obuchowicz AK, Szumera-Cieckiewicz A, Ptaszynski K, Rutynowska-Pronicka O, Madziara E, Tiszler-Cieslik E, Cienciala M, Pietrzak J, Glowacki J, Perek D. Intraspinal mesenchymal chondrosarcoma in a 14-year-old patient: diagnostic and therapeutic problems in relation to the review of literature. J Pediatr Hematol Oncol. 2012;34:188-192.

22. Shakked RJ, Geller DS, Gorlick R, Dorfman HD. Mesenchymal chondrosarcoma: clinicopathologic study of 20 cases. Arch Pathol Lab Med. 2012;136:61-75.

23. Vencio EF, Reeve CM, Unni KK, Nascimento AG. Mesenchymal chondrosarcoma of the jaw bones. Cancer. 1998;82:2350-2355.

24. Warren JL, Klabunde CN, Schrag D, Bach PB, Riley GF. Overview of the SEER-Medicare data: content, research applications, and generalizability to the United States elderly population. Med Care. 2002;40(Suppl):IV-3-18.

25. Xu J, Li D, Xie L, Tang S, Guo W. Mesenchymal chondrosarcoma of bone and soft tissue: a systematic review of 107 patients in the past 20 years. PLoS One. 2015;10:e122216. 